\title{
Light Dark Matter and Dark Radiation
}

\author{
Jae Ho Hed* and C.S. Kim ${ }^{\dagger}$ \\ Department of Physics and IPAP, Yonsei University, Seoul 120-749, Korea
}

\begin{abstract}
Light $(M \leq 20 \mathrm{MeV})$ dark-matter particles freeze out after neutrino decoupling. If the dark-matter particle couples to a neutrino or an electromagnetic plasma, the late time entropy production from dark-matter annihilation can change the neutrino-to-photon temperature ratio, and equally the effective number of neutrinos $N_{\text {eff. }}$. We study the non-equilibrium effects of dark-matter annihilation on the $N_{\text {eff }}$ and the effects by using a thermal equilibrium approximation. Both results are constrained with Planck observations. We demonstrate that the lower bounds of the dark-matter mass and the possibilities of the existence of additional radiation particles are more strongly constrained for dark-matter annihilation process in non-equilibrium.
\end{abstract}

PACS numbers: 95.35.+d, 98.80.Cq

Keywords: dark matter, dark radiation, effective number of neutrinos

*Electronic address: jaeheo1@gmail.com

†Electronic address: cskim@yonsei.co.kr 


\section{INTRODUCTION}

Photons and neutrinos are the lightest particles in the Standard Model (SM) and give the radiation energy density at late times in the early universe. The SM neutrino species contributes three degrees of freedom because there are exactly three neutrino mass eigenstates $\left(\nu_{1}, \nu_{2}, \nu_{3}\right)$ in combinations of the three flavor eigenstates $\left(\nu_{e}, \nu_{\mu}, \nu_{\tau}\right)$ of the weak interaction. The weak interactions that keep neutrinos in thermal contact with the electromagnetic plasma become ineffective around a second after the Big Bang. Neutrinos decouple at a temperature on the order of $2-3 \mathrm{MeV}$ before $e^{ \pm}$pairs annihilate and, thus, do not share in the entropy transfer from $e^{ \pm}$pairs. This causes the neutrino temperature to be less than the photon temperature later. However, neutrino decoupling was not quite complete when $e^{+} e^{-}$annihilation began, so some of the energy and the entropy of photons could transfer to neutrinos. If the dark radiation density is parameterized in terms of the number of effective neutrino species $N_{\text {eff }}$ with the canonical neutrino-to-photon temperature ratio, $N_{\text {eff }}$ increases to slightly more than the three neutrino species, leading to $N_{\mathrm{eff}}^{\mathrm{SM}}=3.046$ [1, 2]. Because the number of effective neutrino species $N_{\text {eff }}$ is precisely predicted in the SM, this can give a robust constraint to any nonstandard physics. For example, new relativistic particles, such as the light sterile neutrino [3] or the Goldstone boson [4] which has a decoupling temperature less than $100 \mathrm{MeV}$, arise in many extensions of the SM, and their existence will contribute to the dark radiation energy density. This scenario is, however, strongly excluded at over the $3 \sigma$ level in the latest Planck analysis [5] unless photons or electrons (positrons) are heated at a later time [6- 9 ]

According to a recent analysis of the cosmic microwave background (CMB) temperature anisotropy by the Planck satellite [5], $N_{\text {eff }}$ was found to be $3.15 \pm 0.23(1 \sigma)$, consistent with the SM prediction. We should recognize that the Hubble constant $\left(H_{0}=67.8 \pm 0.9 \mathrm{kms}^{-1} \mathrm{Mpc}^{-1}\right)$ inferred by Planck is in tension at about $2.4 \sigma$ with the direct measurement of $H_{0}=73.8 \pm 2.4 \mathrm{kms}^{-1} \mathrm{Mpc}^{-1}$ by the HST [10]: larger values of the Hubble constant prefer larger values of $N_{\text {eff }}$. $N_{\text {eff }}$ was not strongly excluded to about the $2 \sigma$ upper limit in the Planck analysis. Additionally, $N_{\text {eff }}$ can be inferred from big bang nucleosynthesis (BBN) considerations [11] at times earlier than recombination because the theoretical expectations for the primordial abundances of light elements depend on $N_{\text {eff. }}$. Recently, two groups announced different results for $N_{\text {eff }}$ determined from an analysis of ${ }^{4} \mathrm{He}$ 
abundance measurements in combination with the D abundance [12, 13]. One group [14] obtained a result consistent with the Planck observation, but the other [15] found a larger value of $N_{\text {eff }} \sim 3.58$. This probe does not have the same resolving power as the Planck satellite. We will use Planck results to constrain the dark radiation in this work.

Recently, weakly interacting massive particles (WIMPs) with sub-GeV masses [16-21], referred to as light dark matter (DM), have received some attention because the existence of WIMPs with mass less than $20 \mathrm{MeV}$ can modify the early universe energy's and entropy densities. If DM particles couple to the SM particles (neutrinos, or photons and $e^{ \pm}$pairs) and are sufficiently light $(M \leq 20 \mathrm{MeV})$ to annihilate after neutrino decoupling, the primordial plasma will be heated by DM annihilation. This will affect the neutrino-to-photon temperature ratio and so might be able to explain possible differences of $N_{\text {eff }}$ from the SM prediction or to avoid strong experimental constraints on the existence of additional radiation particles. This scenario was studied in the equilibrium version for neutrino heating [7, 8, 22] and photon heating [6 9]. The equilibrium version is, however, a rough approximation because DM particles are nonrelativistic at freeze-out ${ }^{1}$. If the DM particles are relativistic at DM decoupling, the equilibrium method must be a good approximation because they will decouple at equilibrium concentrations. A more accurate description of the freeze-out process should be considered when DM particles are nonrelativistic at freeze-out. Here, the Boltzmann equation is applied to the time-evolution of the DM number in a spatially homogeneous and isotropic universe. We treat an adiabatic expansion of the universe so that the total entropy stays constant and the second law of thermodynamics can be applied to the entropy (temperature) evolution of the produced relativistic particles. Because the DM mass $(M)$ determines the dark radiation energy, it will be the parameter that we will constrain. We start to study the DM number evolution for an expanding universe and dark radiation $\left(N_{\text {eff }}\right)$ in the equilibrium approximation. Then, we study the out-of-equilibrium light-particle production, its entropy (temperature) evolution and its effect on the dark radiation. The possibility of the existence of new light species (equivalent neutrinos) is also

\footnotetext{
${ }^{1}$ We distinguish terminology, "decoupling" and "freeze-out", in this paper. "Decoupling" will be used in the case that (DM) particles are completely non-interacting at some point, and "freeze-out" is for chemical decoupling. Notice this does not mean that DM dumps energy in the neutrino or the electromagnetic plasma instantaneously. The evolving comoving number density of DM particles has a sizable deviation from its equilibrium prediction around freeze-out (see Fig. 1).
} 
investigated.

\section{THEORETICAL DETAILS AND NUMERICAL RESULTS}

The dark radiation energy density of the universe $\rho_{\mathrm{DR}}$ is parameterized in terms of the energy density of photons $\rho_{\gamma}$ and the effective number of neutrinos $N_{\text {eff }}$ with the neutrinoto-photon temperature ratio of the SM given by

$$
\frac{\rho_{\mathrm{DR}}}{\rho_{\gamma}}=\frac{7}{8} N_{\mathrm{eff}}\left(\frac{T_{\nu}}{T_{\gamma}}\right)_{\mathrm{SM}}^{4} .
$$

The factor $7 / 8$ is due to the effect of Fermi-Dirac statistics on the energy density. The exact formula for $N_{\text {eff }}$ depends on the scenario (model). Because the temperature will change in our scenario, $N_{\text {eff }}$ can be expressed by

$$
N_{\text {eff }}=N_{\text {eff }}^{\mathrm{SM}}\left(\frac{T_{\nu}}{T_{\gamma}}\right)^{4}\left(\frac{T_{\nu}}{T_{\gamma}}\right)_{\mathrm{SM}}^{-4} .
$$

We have assumed that DM particles are nonrelativistic at the time we consider, for example, the time of DM freeze-out or recombination. The BBN imposes limits on $N_{\text {eff }}$ at photon temperatures around $1-0.1 \mathrm{MeV}$, and any additional dark radiation particle is unfavorable from BBN considerations though a small possibility still exists. If the DM particle is relativistic in the $\mathrm{BBN}$ era, it becomes a dark radiation particle. We investigate the effects of DM annihilation on $N_{\text {eff }}$ for the DM mass range of $0.1-20 \mathrm{MeV}$. The ratio of the neutrino temperature to the photon temperature can be determined by entropy conservation because the total entropy stays constant in an adiabatic expansion of the universe. After neutrino decoupling, the primeval plasma will consist of two decoupled components, the

electromagnetic component and three neutrino ones. The entropies of the neutrino and the electromagnetic plasmas are separately conserved, and this must serve as an efficient tool for the study of dark radiation here.

Two independent thermal baths exist after neutrino decoupling, and we will consider the DM interaction (annihilation) in each thermal bath. Here, the DM particle always interacts with the plasma in thermal bath $a$, and thermal bath $b$ is not relevant to the DM interaction, i.e., $a, b=\nu$ or $\gamma$, but $a \neq b$. 


\section{A. Dark-matter Number Evolution}

DM particles are in thermal contact with the rest of the cosmic plasma at high temperatures, but they will experience the freeze-out at a critical temperature. In this case, we should consider the Boltzmann equation for the evolution of DM number. Because the DM interacts with one of the plasmas, we express the comoving number density about the temperature of the plasma in thermal bath $b$. This notation is very useful because one plasma can always be in the thermal equilibrium. If the DM interacts with the plasma in thermal bath $a$, the evolution equation for the comoving number density $Y\left(\equiv n_{D M} / s_{b}\right)$ with respect to the inverse temperature $x_{b}\left(\equiv M / T_{b}\right)$ of the other thermal bath reads

$$
\frac{d Y}{d x_{b}}=-\frac{\langle\sigma v\rangle s_{b}}{x_{b} H}\left(Y^{2}-Y_{\mathrm{eq}}^{2}\right)
$$

where $H$ is the Hubble parameter, $s_{b}$ is the entropy density in thermal bath $b$ and $Y_{\text {eq }}$ $\left(=n_{e q} / s_{b}\right)$ is the equilibrium number density. This equation is not meaningful when $Y=$ $Y_{\text {eq. }}$. This becomes the usual fluid equation in thermal equilibrium. We parameterize the annihilation cross section as $\langle\sigma v\rangle=\sigma_{0} x_{b}^{-n}$, in which $n=0$ for $s$-wave annihilation and $n=1$ for $p$-wave annihilation. The above equation can be reduced to

$$
\frac{d Y}{d x_{b}}=-\sqrt{\frac{\pi}{45}} m_{\mathrm{PL}} M \sigma_{0}\left(\frac{g_{* s}^{b}}{\sqrt{g_{*}}}\right) x_{b}^{-n-2}\left(Y^{2}-Y_{\mathrm{eq}}^{2}\right),
$$

where $g_{*}$ and $g_{* s}^{b}$ are the effective relativistic degrees of freedom for the energy density and the entropy respectively, and $m_{\mathrm{PL}}$ is the Planck mass.

Unfortunately, Eq. (4) has no analytic solution. Fig. 1 shows the result of numerical solutions for $s$-wave annihilation into neutrinos (left panel) and $p$-wave annihilation into photons (right panel). The DM residual annihilation into photons can distort the CMB spectrum [23, 24]. This effect excludes DMs with mass less than $10 \mathrm{GeV}$ for $s$-wave annihilation into photons. The effect is negligible for $p$-wave annihilation, which is velocity dependent, so this bound can be evaded. For DM annihilation into neutrinos, we assumed that the same numbers of neutrinos and antineutrinos of each type were produced. The number of effective relativistic degrees of freedom $g_{* s}^{b}$ is not related to DM or its production. We could take the value on the SM base. The $g_{*}$ is taken as a constant $\bar{g}_{*}$ on average. The

curves were made with the proper values of $\sigma_{0} \bar{g}_{*}^{-1 / 2}$, in agreement with the current DM relic density $\left(Y_{0}\right)$ for a Dirac fermion, Majorana fermion, complex scalar and real scalar with a 

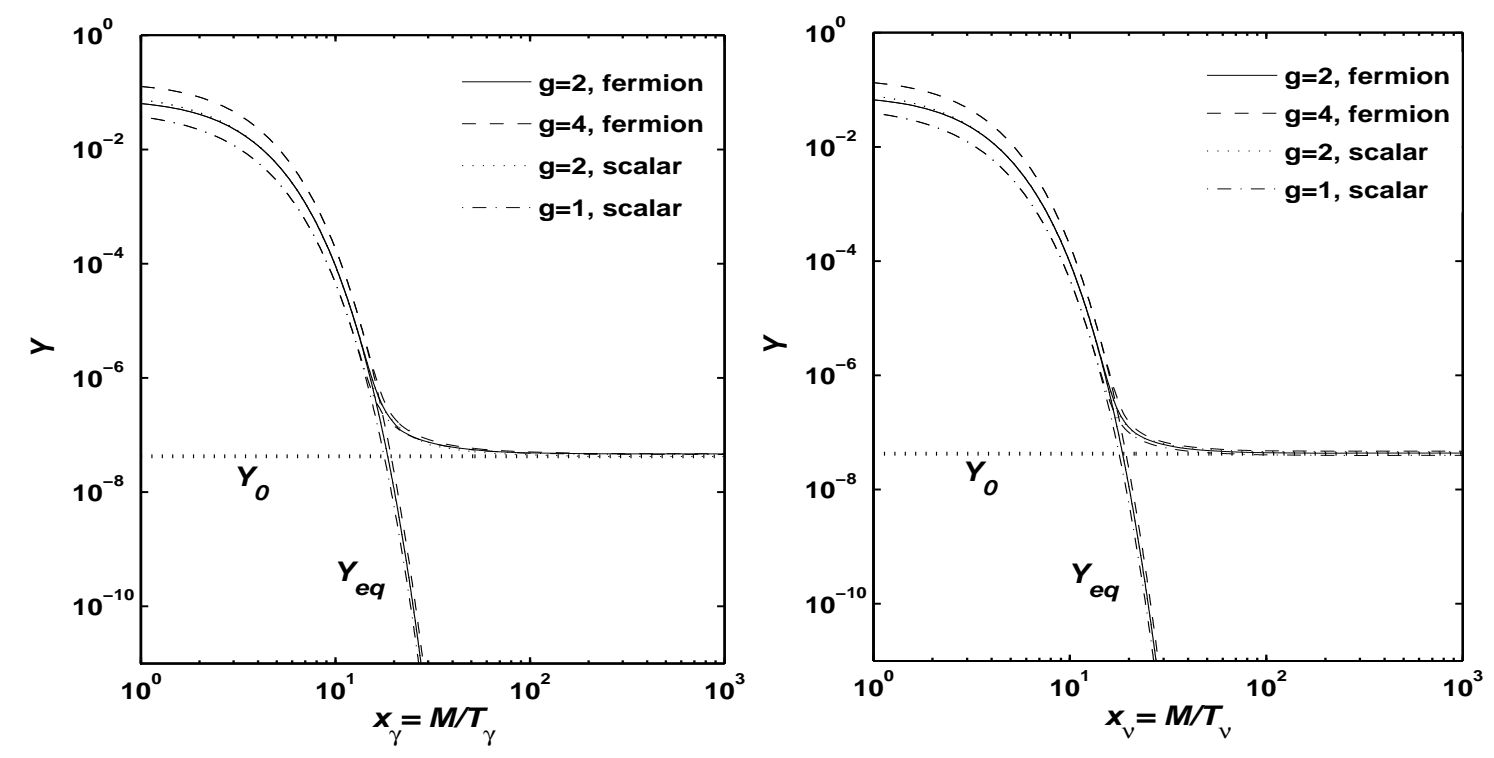

FIG. 1: Comoving number density $Y$ as a function of inverse temperature $x_{\gamma}\left(=M / T_{\gamma}\right)$ and $x_{\nu}\left(=M / T_{\nu}\right)$ for a $g=1$ real scalar (short dash), $g=2$ Majorana (solid), $g=2$ complex scalar (dotted) and $g=4$ Dirac dark matter (long dash) with a DM mass of $10 \mathrm{MeV}$. The left panel is for $s$-wave annihilation into neutrinos with $\sigma_{0} \bar{g}_{*}^{-1 / 2}=(2.8-3.0) \times 10^{-26} \mathrm{~cm}^{3} / \mathrm{s}$, and the right panel is for $p$-wave annihilation into photons with $\sigma_{0} \bar{g}_{*}^{-1 / 2}=8.0 \times 10^{-25} \mathrm{~cm}^{3} / \mathrm{s}$. The horizontal dotted line $\left(Y_{0}\right)$ represents the current DM relic density, and $Y_{\text {eq }}$ indicates the equilibrium number density.

DM mass of $10 \mathrm{MeV}$. As we expected, the DM number track the equilibrium abundance at very high temperatures, $x_{b}<1$. The solution to the Boltzmann equation starts to deviate significantly from the equilibrium abundance at around $x_{b} \sim 10-11$, and the actual DM abundance $Y$ is different from $Y_{\text {eq }}$ and $Y_{0}$ for a considerable time. Notice that the equilibrium number densities at high tenperatures (early times) are different for different particle species. Because the current DM relic density is not relalted to the particle species, a large number of relativisitic particles will be produced for the species that has a large equilibrium number density at early times. This can be a means to distinguish the nature of the particle if other information about the DM, such as the DM mass, is given (see Figs. 2 and 3). 


\section{B. Thermal Equilibrium Approximation}

DM particles are assumed to keep in thermal contact with one of plasmas after neutrino decoupling and to decouple suddenly at some point. The DM and its products can be expressed by Fermi-Dirac or Bose-Einstein statistics in this case. There can be different types of particles in thermal bath $a$, so we will use the entropy density $s_{a} \equiv \frac{2 \pi^{2}}{45} \widetilde{g}_{* s}^{a} T_{a}^{3}=\left(\rho_{a}+p_{a}\right) / T_{a}$ to define the number of effective relativistic degrees of freedom ${ }^{2} \widetilde{g}_{* s}^{a}\left(T_{a}\right)$ in which $\rho_{a}$ is the energy density and $p_{a}$ is the pressure. The energy density $\rho_{a}$ and the pressure $p_{a}$ are expressed by

$$
\begin{aligned}
\rho_{a} & =\sum_{i} \rho_{i}=\sum_{i} \frac{g_{i}}{2 \pi^{2}} \int d q q^{2} E_{i} \frac{1}{\exp \left(E_{i} / T_{a}\right) \pm 1} \\
p_{a} & =\sum_{i} p_{i}=\sum_{i} \frac{g_{i}}{2 \pi^{2}} \int d q \frac{q^{4}}{3 E_{i}} \frac{1}{\exp \left(E_{i} / T_{a}\right) \pm 1}
\end{aligned}
$$

where $g_{i}$ is the internal degrees of freedom for the corresponding particle $i, E_{i}=\sqrt{q^{2}+m_{i}^{2}}$ is the energy with mass $m_{i}$, and the $+(-)$ sign is for fermions (bosons). We set the chemical potentials to zero. The number of effective relativistic degrees of freedom is given by

$$
\widetilde{g}_{* s}^{a}\left(T_{a}\right)=\frac{45}{2 \pi^{2}} \frac{\left(\rho_{a}+p_{a}\right)}{T_{a}^{4}}
$$

Because the entropy in each thermal bath is conserved after neutrino decoupling, the tem-

perature $T_{a}\left(T_{b}\right)$ varies as $\widetilde{g}_{* s}^{a-1 / 3} R^{-1}\left(g_{* s}^{b-1 / 3} R^{-1}\right)$, where $R$ is the scale factor. We can find the temperature ratio at the DM decoupling time if we know the temperature ratio at a certain time (the time of neutrino decoupling). The temperature ratio at the time of DM decoupling results in

$$
\frac{T_{a D}}{T_{b D}}=\left(\frac{\widetilde{g}_{* s}^{a}\left(T_{\nu d}\right)}{\widetilde{g}_{* s}^{a}\left(T_{a D}\right)}\right)^{1 / 3}\left(\frac{g_{* s}^{b}\left(T_{b D}\right)}{g_{* s}^{b}\left(T_{\nu d}\right)}\right)^{1 / 3},
$$

where $T_{\nu d}$ is the neutrino decoupling temperature at which the photon and the neutrino temperatures are the same, $T_{a D}$ and $T_{b D}$ are temperatures ${ }^{3}$ at DM decoupling. This formula can be approximated to the temperature ratio at late times; i.e., at the times when the temperatures $T_{a}, T_{b}$ are less than the decoupling temperatures. Because the DM decoupling

\footnotetext{
${ }^{2}$ The mark " " is placed on top of the symbol of the number of the effective relativistic degrees of freedom to indicate DM inclusion. If there is no " $\sim$ " mark, DM is excluded.

${ }^{3}$ Notice that one of the DM decoupling temperatures is determined when the equilibrium DM number is the same as the present-day DM relic density, $Y_{e q}\left(T_{b D}\right)=Y_{0}$.
} 
TABLE I: $1 \sigma$ and $2 \sigma$ lower limits on the dark-matter mass and upper limits on the existence of any other dark radiation for dark matter in thermal equilibrium with neutrinos or electromagnetic plasmas. The mark '-' indicates that the limit is irrelevant. The symbol "S" stands for scalar and "F" for fermion.

\begin{tabular}{c|ccc|ccc|cccc}
\hline \hline & \multicolumn{3}{|c|}{ Neutrino-coupled DM (MeV) } & \multicolumn{3}{|c}{ EM-coupled DM $(\mathrm{MeV})$} & \multicolumn{4}{|c}{$\Delta N_{\text {eff }}$} \\
\hline$g$ & $1(\mathrm{~S})$ & $2(\mathrm{~S}, \mathrm{~F})$ & $4(\mathrm{~F})$ & $1(\mathrm{~S})$ & $2(\mathrm{~S}, \mathrm{~F})$ & $4(\mathrm{~F})$ & $1(\mathrm{~S})$ & $2(\mathrm{~S})$ & $2(\mathrm{~F})$ & $4(\mathrm{~F})$ \\
\hline $1 \sigma$ & 6.4 & 9.3 & 11.9 & 10.1 & 12.6 & 14.9 & 0.94 & 1.37 & 1.27 & 1.80 \\
$2 \sigma$ & 3.7 & 7.1 & 10.0 & - & - & - & 1.17 & 1.60 & 1.50 & 2.03 \\
\hline \hline
\end{tabular}

occurs at $x_{D} \sim 18$ as we saw in the subsection A, there must be almost no DM contribution to the relativistic degrees of freedom $\widetilde{g}_{* s}^{a}\left(T_{a D}\right)$. We remove the mark " $\sim$ ". The temperature ratio after DM decoupling is then given by

$$
\frac{T_{a}}{T_{b}} \simeq\left(\frac{\widetilde{g}_{* s}^{a}\left(T_{\nu d}\right)}{g_{* s}^{a}\left(T_{a}\right)}\right)^{1 / 3}\left(\frac{g_{* s}^{b}\left(T_{b}\right)}{g_{* s}^{b}\left(T_{\nu d}\right)}\right)^{1 / 3}
$$

We now determine $N_{\text {eff }}$ in each case. If the DM particle interacts with a neutrino $(a=\nu$ and $b=\gamma$ ), the electromagnetic plasma is not relevant to the DM interaction. We can identify $\left(g_{* s}^{\gamma}\left(T_{\gamma}\right) / g_{* s}^{\gamma}\left(T_{\nu d}\right)\right)^{1 / 3}$ with $\left(T_{\nu} / T_{\gamma}\right)_{\mathrm{SM}}$. We get the effective number of neutrino species from Eqs. (2) and (9)

$$
N_{\mathrm{eff}}^{\nu}=N_{\mathrm{eff}}^{\mathrm{SM}}\left(\frac{\widetilde{g}_{* s}^{\nu}\left(T_{\nu d}\right)}{g_{* S}^{\nu}\left(T_{\nu}\right)}\right)^{4 / 3}
$$

If the DM particle interacts with an eletromagnetic plasma $(a=\gamma$ and $b=\nu)$, there is only one species in the neutrino thermal bath, $\nu$. The effective relativistic degrees of freedom $g_{* s}^{\nu}\left(T_{\nu}\right)$ will be the same at any time. Because $\left(T_{\nu} / T_{\gamma}\right)_{\mathrm{SM}}=\left(g_{* s}^{\gamma}\left(T_{\gamma}\right) / g_{* s}^{\gamma}\left(T_{\nu d}\right)\right)^{1 / 3}$, we get the effective number of neutrino species as

$$
N_{\mathrm{eff}}^{\gamma}=N_{\mathrm{eff}}^{\mathrm{SM}}\left(\frac{g_{* s}^{\gamma}\left(T_{\nu d}\right)}{\widetilde{g}_{* s}^{\gamma}\left(T_{\nu d}\right)}\right)^{4 / 3}
$$

The curves of Fig. 2 display numerical results for the $N_{\text {eff }}-M$ relation for a Dirac fermion, Majorana fermion, complex scalar and real scalar. The upper (lower) set of curves are for the case when DM particles are interacting with neutrinos (electrons and photons). We have implicitly assumed that neutrinos decouple at $T_{\nu d} \approx 2.3 \mathrm{MeV}[25-27] . N_{\text {eff }}$ increases for lighter DM if they are in equilibrium with neutrinos. Conversely, $N_{\text {eff }}$ decreases for lighter 


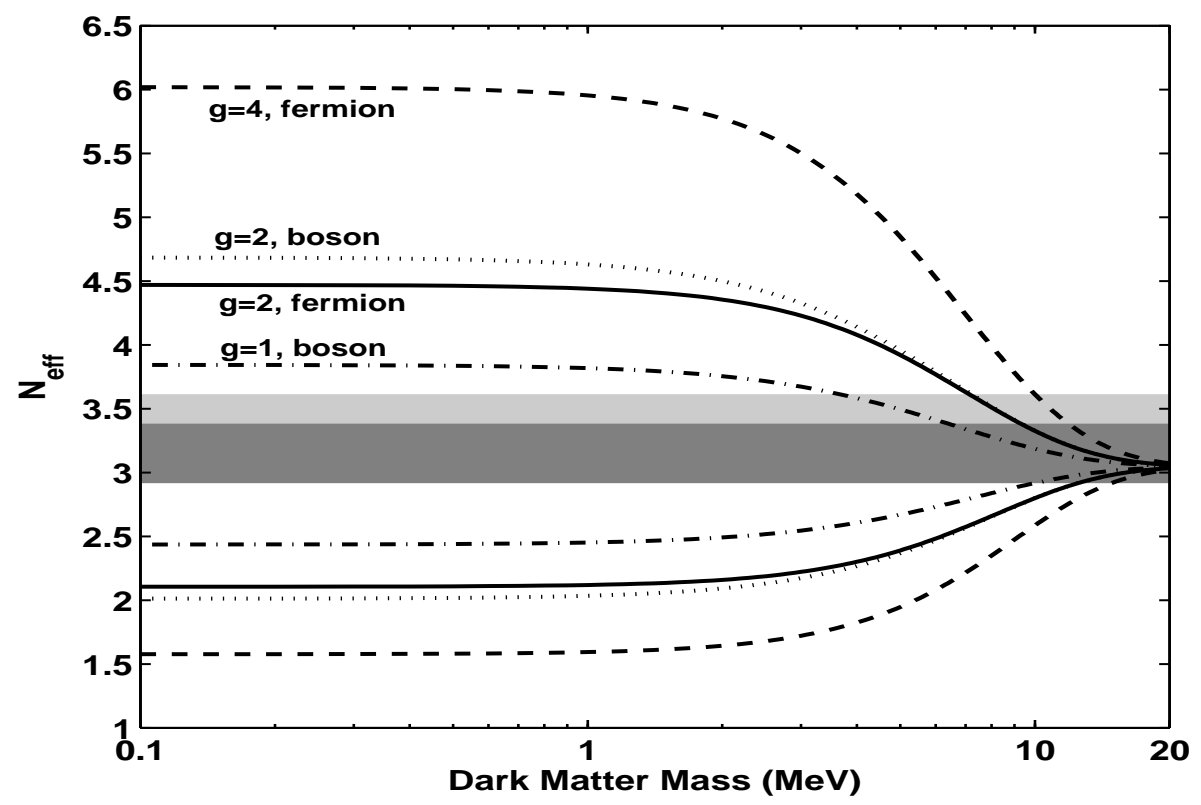

FIG. 2: Effective number of neutrino degrees of freedom, $N_{\text {eff }}$, as a function of a thermal darkmatter mass $M$. Curves correspond to a $g=1$ self-conjugate scalar (short dash), $g=2$ Majorana (solid), $g=2$ complex scalar (dotted) and $g=4$ Dirac dark matter (long dash). The upper (lower) curves are for the case when the dark-matter particles are in thermal equilibrium with neutrinos (electrons and photons). The dark horizontal band is the Planck CMB $1 \sigma$ allowed range, and the light dark band is the $2 \sigma$ upper allowed range.

DM in equilibrium with an electromagnetic plasma. We put a bound on the DM mass by requiring that $N_{\text {eff }}$ be compatible with the measured value from Planck [5], and the bounds of the DM masses are listed in Table I for each species. If there is a significant, but small, density of additional radiation, the additional radiation density can be explained by neutrino heating from DM annihilation. We should notice that there is still enough room for the existence of additional radiation particles $\left(\Delta N_{\text {eff }}\right)$, such as a sterile neutrino or a Goldstone boson, with decoupling temperatures less than $100 \mathrm{MeV}$, when DM electromagnetically couples to SM particles (EM-coupled DM). 


\section{Out-of-equilibrium Production}

As we can see in Fig. 1, there is a smooth transition between two regions, before and after DM freeze-out, and DM particles do not track significantly the equilibrium from $x_{b} \sim 10-11$. The second law of thermodynamics is applied to the entropy (temperature) evolution of the produced relativistic particles:

$$
d S_{a}=\frac{d Q}{T_{b}},
$$

where $d Q=d\left(R^{3} \rho_{\mathrm{DM}}\right)$ is the heat added per comoving volume due to DM annihilation. Because the number of DM particles is reduced by their annihilation at temperature smaller than DM mass, the energy density of DM can be described in its nonrelativistic approximation, $\rho_{\mathrm{DM}} \simeq n_{\mathrm{DM}} M=M s_{b} Y$. The change in entropy ${ }^{4}$ is given by

$$
d S_{a}=-S_{b} x_{b} d Y \longrightarrow \Delta S_{a}=-S_{b} \int_{i} x_{b} d Y
$$

where $i$ is an initial point. We consider the initial point at the time of neutrino decoupling because it is the last point at which neutrinos and photons are in thermal contact. DM particles with masses less than $20 \mathrm{MeV}$ must be in thermal equilibrium in thermal bath $a$ at the initial point. Our observational point is the time of recombination, much later after freeze-out. After freeze-out (chemical decoupling), DM continues to scatter off relativistic SM particles untill DM kinetic decoupling, thus thermalizing the produced particles. Produced electrons (positrons) or photons will be thermalized quickly due to the electromagnetic interaction. Thermalization of neutrinos must be slow. Because neutrinos continue to scatter off DM particles after freeze-out, the produced neutrinos ${ }^{5}$ can be in the equilibrium at recombination. The change in entropy is expressed by

$$
\Delta S_{a}=S_{a}-S_{a i}=\frac{2 \pi^{2}}{45}\left[g_{* s}^{a} T_{a}^{3} R^{3}-\left(g_{* s}^{a} T_{a}^{3} R^{3}\right)_{i}\right]
$$

\footnotetext{
4 The plasma in the thermal bath $b$ is always in thermal equilibrium because it is not relevant to the DM interaction, so the entropy $S_{b}$ is constant.

${ }^{5}$ A certain number of neutrinos can remain in non-equilibrium if their scattering strength is not enough large. We need to consider the detailed Boltzmann equation with the scattering cross section for this process. Because our work is not concerned with any specific model, we assume that the produced neutrinos are in the equilibrium at recombination. The details with scattering are left for a future study.
} 


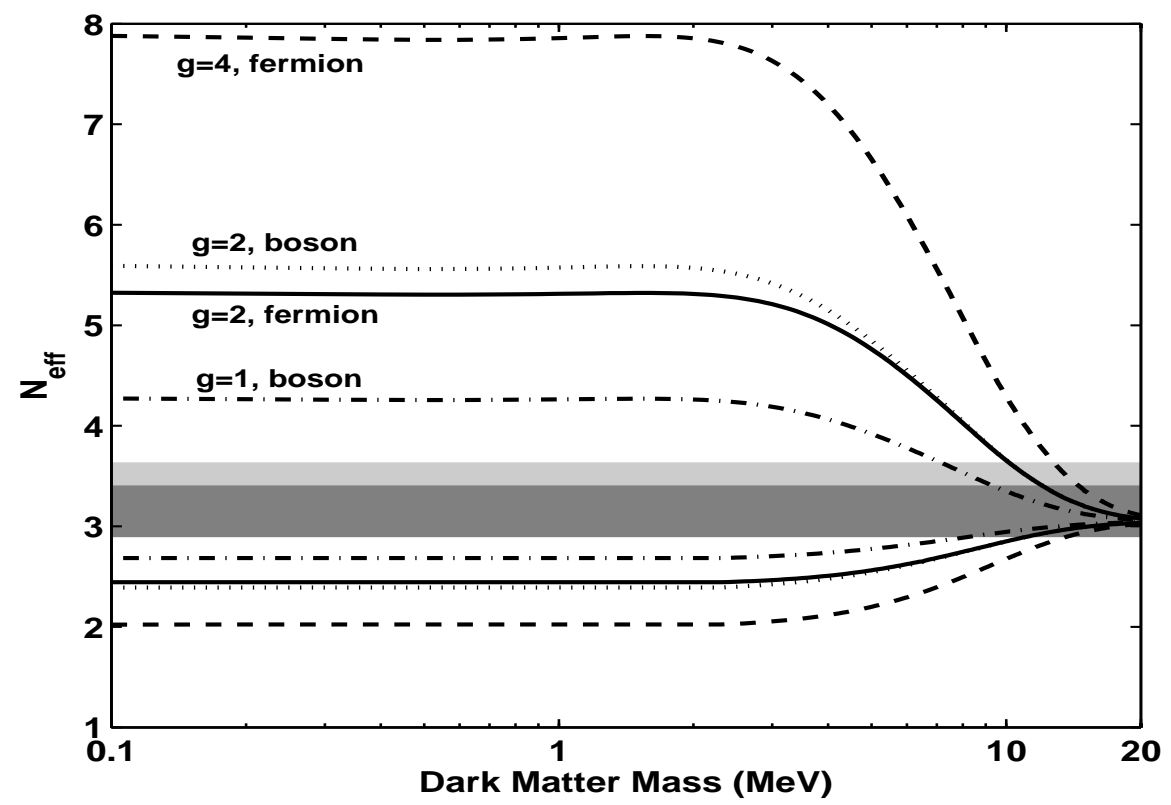

FIG. 3: Same as Fig. 2, but contour lines are for the case when radiation particles are produced from dark-matter annihilation in the non-equilibrium method (freeze-out mechanism).

The temperature ratio is determined by a combination of Eqs. (13) and (14):

$$
\left(\frac{T_{a}}{T_{b}}\right)^{3}=\left(\frac{g_{* s i}^{a}}{g_{* s}^{a}}\right)\left(\frac{R_{i}}{R}\right)^{3}\left(\frac{T_{a i}}{T_{b}}\right)^{3}-\frac{g_{* s}^{b}}{g_{* s}^{a}} \int_{i} x_{b} d Y,
$$

where $T_{a i}$ is, according to Ref. [6], very similar to the neutrino decoupling temperature described in the SM of the absence of DM. Using the entropy conservation $\left(g_{* s}^{a} R^{3} \sim T^{-3}\right)$ in the SM, we can approximate the first term of Eq. (15). The temperature ratio is given by

$$
\left(\frac{T_{a}}{T_{b}}\right)^{3} \simeq\left(\frac{T_{a}}{T_{b}}\right)_{S M}^{3}-\frac{g_{* s}^{b}}{g_{* s}^{a}}\left[x_{b} Y-\left(x_{b} Y\right)_{i}-\int_{i} Y d x_{b}\right],
$$

where we have introduced the integration method by parts, as a convenience, for numerical computations. The first term on the right-hand side of Eq. (16) is just the original temperature ratio in radiation, and the second term represents a contribution from DM annihilation. We can express the temperature ratio in each case, neutrino-coupled DM $(a=\nu$ and $b=\gamma)$ and EM-coupled DM $(a=\gamma$ and $b=\nu)$.

Fig 3 shows the numerical results of the $N_{\text {eff }}-M$ relation for neutrino-coupled DM, the upper set of curves, and EM-coupled DM, the lower set curves. The basic arguments are the same as those in the equilibrium approximation of subsection B. The bounds on 
TABLE II: Same as Table. I, but the values are for the case when radiation particles are produced from dark-matter annihilation in the non-equilibrium method (freeze-out mechanism).

\begin{tabular}{c|ccc|ccc|cccc}
\hline \hline & \multicolumn{3}{|c|}{ Neutrino-coupled DM (MeV) } & \multicolumn{3}{|c}{ EM-coupled DM $(\mathrm{MeV})$} & \multicolumn{4}{|c}{$\Delta N_{\text {eff }}$} \\
\hline$g$ & $1(\mathrm{~S})$ & $2(\mathrm{~S}, \mathrm{~F})$ & $4(\mathrm{~F})$ & $1(\mathrm{~S})$ & $2(\mathrm{~S}, \mathrm{~F})$ & $4(\mathrm{~F})$ & $1(\mathrm{~S})$ & $2(\mathrm{~S})$ & $2(\mathrm{~F})$ & $4(\mathrm{~F})$ \\
\hline $1 \sigma$ & 9.6 & 12.3 & 14.8 & 9.1 & 11.8 & 14.3 & 0.70 & 0.99 & 0.94 & 1.36 \\
$2 \sigma$ & 7.3 & 10.3 & 12.9 & - & - & - & 0.93 & 1.22 & 1.16 & 1.56 \\
\hline \hline
\end{tabular}

the DM masses are also listed in Table II, as well as possibilities for the existence of additional radiation particles $\left(\Delta N_{\text {eff }}\right)$. In this DM annihilation process, the DM mass bounds are more stringent, and the DM effect on the existence of additional radiation particles is stronger. We interpret this in the following way: DM particles annihilate more slowly into SM particles to make a smooth transition. In the radiation-dominant era, $H=$ $(1 / R) d R / d t \simeq \sqrt{(8 / 3) \pi G \rho_{\mathrm{R}}}$, with the gravitational constant $G=1 / m_{\mathrm{PL}}^{2}$ and the radiation energy density $\rho_{\mathrm{R}}$. The slower annihilation results in a smaller expansion of the universe and eventually a smaller size of the universe later. The same number of relativistic particles must be produced from DM annihilation in the equilibrium and the non-equilibrium processes. The predicted energy densities at a later time are larger than they are in the equilibrium process, so DM annihilation effects are larger in the non-equilibrium process.

\section{CONCLUSIONS}

Light $(M \leq 20 \mathrm{MeV})$ dark-matter particles freeze out after neutrino decoupling. If the dark-matter particle interacts with a neutrino or an electromagnetic plasma, the latetime entropy production from dark-matter annihilation can change the neutrino-to-photon temperature ratio, and equally the effective number of neutrinos $N_{\text {eff. }}$ We studied the effects of dark-matter annihilation on the $N_{\text {eff }}$ by using the thermal equilibrium approximation and non-equilibrium method (freeze-out mechanism), and both results were compared with Planck observations. If a significant, but small, density of additional radiation exists, this can be explained by neutrino heating from dark-matter annihilation. The effective number of neutrino species $N_{\text {eff }}$ is reduced for photon heating. In that case, the existence of additional dark radiation particles can help improve the agreement with the current observations. The 
dark-matter particles annihilate more slowly into SM particles for dark matter annihilation in non-equilibrium. The slower annihilation results in a smaller expansion rate (eventually a smaller universe later). Although the same number of relativistic particles are produced from dark-matter annihilation in the equilibrium approximation and the non-equilibrium method, the predicted energy densities at a later time are different. We demonstrated that the lower bounds on the dark-matter mass and the possibilities of the existence of additional radiation particles are more strongly constrained for dark-matter annihilation process in non-equilibrium.

\section{Acknowledgments}

The work is supported by a National Research Foundation's Korea (NRF) grant funded by Korea government of the Ministry of Education, Science and Technology (MEST) (Grant No. 2011-0017430 and Grant No. 2011-0020333).

[1] D. A. Dicus, E. W. Kolb, A. M. Gleeson, E. C. G. Sudarshan, V. L. Teplitz, and M. S. Turner, Phys. Rev. D 26, 2694 (1982).

[2] G. Mangano, G. Miele, S. Pastor, and M. Peloso, Phys. Lett. B 534, 8 (2002), astro-ph/0111408

[3] K. N. Abazajian, M. A. Acero, and S. K. Agarwalla, et al., arXiv:1204.5379.

[4] S. Weinberg, Phys. Rev. Lett. 110, 241301 (2013), arXiv:1305.1971.

[5] P. A. R. Ade, et al. [Planck Collaboration], arXiv:1502.01589.

[6] C. M. Ho, and R. J. Scherrer, Phys. Rev. D 87, 023505 (2013), arXiv:1208.4347; Phys. Rev. D 87, 065016 (2013), arXiv:1212.1689.

[7] G. Steigman, Phys. Rev. D 87, 103517 (2013), arXiv:1303.0049.

[8] C. Boehm, M. J.Dolan, and C. McCabe, J. Cosmol. Astropart. Phys. 08, 041 (2013), arXiv:1303.6270.

[9] K. M. Nollett, and G. Steigman, Phys. Rev. D 89, 083508 (2014), arXiv:1312.5725; Phys. Rev. D 91, 083505 (2015), arXiv:1411.6005.

[10] A. G. Riess, L. Macri, S. Casertano, H. Lampeitl, H. C. Ferguson, A. V. Filippenko, S. 
W. Jha, and W. Li, et al., Astrophys. J. 730, 119 (2011) [Erratum-ibid. 732, 129 (2011)], arXiv:1103.2976.

[11] J. Yang, D. Schramm, G. Steigman, and R. T. Rood, Astrophys. J. 227, 697 (1979).

[12] R. Cooke, M. Pettini, R. A. Jorgenson, M. T. Murphy, and C. C. Steidel, Astrophys. J. 781, 31 (2014), arXiv:1308.3240.

[13] M. Pettini, and R. Cooke, Mon. Not. R. Astron. Soc. 425, 2477 (2012), arXiv:1205.3785.

[14] E. Aver, K. A. Porter, R. L. Porter, and E. D. Skillman, J. Cosmol. Astropart. Phys. 11, 017 (2013), arXiv:1309.0047.

[15] Y. I. Izotov, T. X. Thuan, and N. G. Guseva, Mon. Not. Roy. Astron. Soc. 445, 778 (2014), arXiv:1408.6953.

[16] E. W. Kolb, M. S. Turner, and T. P. Walker, Phys. Rev. D 34, 2197 (1986).

[17] P. D. Serpico, and G. G. Raffelt, Phys. Rev. D 70, 043526 (2004), astro-ph/0403417.

[18] C. Boehm, and P. Fayet, Nucl. Phys. B 683, 219 (2004), hep-ph/0305261.

[19] C. Boehm, D. Hooper, J. Silk, M. Casse, and J. Paul, Phys. Rev. Lett. 92, 101301 (2004), astro-ph/0309686

[20] D. Hooper, F. Ferrer, C. Boehm, J. Silk, J. Paul, N. W. Evans, and M. Casse, Phys. Rev. Lett. 93, 161302 (2004), astro-ph/0311150.

[21] K. Ahn, and E. Komatsu, Phys. Rev. D 72, 061301 (2005), astro-ph/0506520.

[22] C. Boehm, M. J. Dolan, and C. McCabe, J. Cosmol. Astropart. Phys. 12, 027 (2012), arXiv:1207.0497.

[23] D. P. Finkbeiner, S. Galli, T. Lin, and T. R. Slatyer, Phys. Rev. D 85, 043522 (2012), arXiv:1109.6322.

[24] L. Lopez-Honorez, O. Mena, S. Palomares-Ruiz, and A. C. Vincent, J. Cosmol. Astropart. Phys. 07, 046 (2013), arXiv:1303.5094.

[25] K. Enqvist, K. Kainulainen, V. Semikoz, Nucl. Phys. B 374, 392 (1992).

[26] A. D. Dolgov, Phys. Rept. 370, 333 (2002), hep-ph/0202122.

[27] S. Hannestad, Phys. Rev. D 65, 083006 (2002), astro-ph/0111423. 